\title{
Extreme Wave Events in Directional, Random Oceanic Sea States
}

\author{
Submitted
}

\author{
M. Onorato, A. R. Osborne and M. Serio \\ Dipartimento di Fisica Generale, Universitá di Torino, Torino, Via P. Giuria, 1 - 10125, ITALY
}

\begin{abstract}
We discuss the effects of the directional spreading on the occurrence of extreme wave events. We numerically integrate the envelope equation recently proposed by Trulsen et al., Phys of Fluids 2000, as a weakly nonlinear model for realistic oceanic gravity waves. Initial conditions for numerical simulations are characterized by the spatial JONSWAP power spectrum for several values of the significant wave height, steepness and directional spreading. We show that by increasing the directionality of the initial spectrum the appearance of extreme events is notably reduced.
\end{abstract}

We address the occurrence of extreme wave events in numerical models of random, directional, oceanic sea states. Extreme ocean waves of this type are of unusually large size with respect to the background, surrounding waves. They are often refered to as "freak" or "rogue" waves and have been given a rough definition in terms of an arbitrary threshold, $H_{\max }>2.2 H_{s}$, for $H_{s}$ the significant wave height [1]. Because extreme waves are rare, and hence are not often measured, the physical mechanisms for their occurrence have not been unequivocally established. Possible causes of freak waves in the open sea have been investigated by many researchers [2] and basically three mechanisms have been proposed: the linear interaction of the waves with the currents (geometrical optic theory), see 3 . 5]; the simple linear superposition of Fourier phases (the resulting large waves are also known as transient waves [6]) and the modulation instability [7].

The last mechanism and indeed the method addressed herein is based on the nonlinear phenomenon known as the Benjamin-Feir instability [8], which describes how a monochromatic wave train can be unstable to small, side-band perturbations. This physical phenomena has been widely studied in wave tank facilities (see for example [9], [10] and references therein) and from a theoretical and numerical point of view for the $1+1$ Nonlinear Schroedinger equation (NLS equation in one space and one time dimensions) 11. 12 and for the fully nonlinear Euler equations [13]. A major complication arises from the fact the envelope equations (for example NLS) are derived from the primitive equations of motion under the hypothesis of a narrow-banded spectrum. Higher order equations in the envelope hierarchy have then been proposed [15,16] in order to overcome the limitation of narrow bandwidth. Numerical simulations of these equations in $1+1$ dimension 14, 17] have shown that the probability of occurrence of freak waves is strictly related to the "enhancement" coefficient $\gamma$ and the Phillips parameter $\alpha$ of the JONSWAP power spectrum [18]. In [14] 17] it was found that increasing the values of $\gamma$ and $\alpha$ has the effect of increasing the probability of finding freak wave events. In addition to the physical limitations associated with the narrow spectral width assumption, an even more severe limitation of the above results arises because these conclusions have been obtained from es- sentially one-dimensional numerical simulations.

In this communication we address the less restricted problem of the appearance of freak waves in $2+1$ dimensions, i.e. the wave field depends on the spatial coordinates $x, y$ and time, $t$, so that directional spreading is systematically included. Preliminary results have already been considered in [19] where simple initial conditions characterized by a carrier wave plus longitudinal and transverse perturbations showed an ubiquitous formation of large amplitude "freak" waves under the evolution of the NLS equation in $2+1$. Our aim here, instead, is to study the more realistic case characterized by the JONSWAP spectrum for a directional wave train, while at the same time extending the order of the simulations so that energy leakage to high wave numbers does not occur (see [9]). Recently Trulsen et al. 21] proposed a model equation (see eqs. (4.5) below) that shares many of the properties of the Zakharov equation 20. The main advantage of the model is that it is numerically inexpensive and therefore suitable for extensive numerical simulations in which large amounts of statistical information is desired. The equation can be thought of as a special case of the Zakharov equation:

$$
\begin{aligned}
& i \frac{\partial B(\mathbf{k}, t)}{\partial t}=\int_{-\infty}^{+\infty} T_{\mathbf{k}, \mathbf{k}_{1}, \mathbf{k}_{2}, \mathbf{k}_{3} B\left(\mathbf{k}_{1}, t\right)^{*} B\left(\mathbf{k}_{2}, t\right) B\left(\mathbf{k}_{3}, t\right) \times} \\
& \delta\left(\mathbf{k}+\mathbf{k}_{1}-\mathbf{k}_{2}-\mathbf{k}_{3}\right) e^{i\left(\omega+\omega_{1}-\omega_{2}-\omega_{3}\right) t} d \mathbf{k}_{1} d \mathbf{k}_{2} d \mathbf{k}_{3},
\end{aligned}
$$

where $T_{\mathbf{k}, \mathbf{k}_{1}, \mathbf{k}_{2}, \mathbf{k}_{3}}=T\left(\mathbf{k}, \mathbf{k}_{1}, \mathbf{k}_{2}, \mathbf{k}_{3}\right)$ is the coupling coefficient (for the analytical form of it see for example [22). We briefly outline the connection of the Zakharov equation with the proposed model. One performs the following change of variables: $\mathbf{k}=\mathbf{k}_{\mathbf{0}}+\chi$ with $\chi=(\lambda, \mu)$ and

$$
A(\boldsymbol{\chi}, t)=B(\mathbf{k}, t) e^{-i\left(\omega(\mathbf{k})-\omega\left(\mathbf{k}_{\mathbf{0}}\right)\right) t}
$$

For simplicity we perform a simple rotation so that $\mathbf{k}_{0}$ has the coordinates $\left(k_{0}, 0\right)$. The next step consists in a Taylor's expansion of the coupling coefficient, $T\left(\mathbf{k}, \mathbf{k}_{1}, \mathbf{k}_{2}, \mathbf{k}_{3}\right)$, up to first order in spectral width around the carrier wave number, $\mathbf{k}_{0}$. Note that the expansion is performed on the nonlinear term, while leaving the linear part of the equation unchanged. As a result, the following particular kernel is used in eq. (11) (see [23] ): 


$$
\begin{array}{r}
T\left(\mathbf{k}_{\mathbf{0}}+\chi, \mathbf{k}_{\mathbf{0}}+\chi_{1}, \mathbf{k}_{\mathbf{0}}+\chi_{2}, \mathbf{k}_{\mathbf{0}}+\chi_{3}\right)=\frac{k_{0}^{3}}{4 \pi^{2}}(1+ \\
\left.\frac{3}{2 k_{0}}\left(\lambda_{2}+\lambda_{3}\right)-\frac{\left(\lambda_{1}-\lambda_{2}\right)^{2}}{2 k_{0}\left|\chi_{1}-\chi_{2}\right|}-\frac{\left(\lambda_{1}-\lambda_{3}\right)^{2}}{2 k_{0}\left|\chi_{1}-\chi_{3}\right|}\right)
\end{array}
$$

Under these conditions the triple integral in eq. (11) can be performed (see [23] for details) and the following set of equations is obtained in physical (configuration) space:

$A_{t}+\omega_{0} L\left(\partial_{x}, \partial_{y}\right) A+i \frac{\omega_{0} k_{0}^{2}}{2}|A|^{2} A+\frac{3 \omega_{0} k_{0}}{2}|A|^{2} A_{x}-$

$\frac{\omega_{0} k_{0}}{4} A^{2} A_{x}^{*}+\left.i k_{0} A \bar{\phi}_{x}\right|_{z=0}=0$

where $L=i\left[\left(\left(1-i \partial_{x}\right)^{2}-i\left(\partial_{y}\right)^{2}\right)^{1 / 4}-1\right]$; use of this latter operator insures retention of linear dispersion to all orders. $\bar{\phi}$ is the inducead mean flow which is coupled with the envelope $A$ via the following nonlocal relation:

$$
\left.\bar{\phi}_{x}\right|_{z=0}=-\frac{\omega_{0}}{2} F^{-1}\left\{|k| F\left\{|A|^{2}\right\}\right\},
$$

with $F$ the Fourier operator. We call equations (45) the Generalized Dysthe Equation (GDE) since the nonlinear part is exactly that of the Dysthe Equation [15], but further includes the linear dispersion relation of the inviscid primitive equations of motion. As stated in 21], this equation shares many properties of the Zakharov equation: the four wave resonant interaction curve coincides with the Phillips curve and moreover the instability region is bounded and is quantitatively similar to the results obtained by McLean 24] for the Zakharov equation (see Fig. 1 in $[21]$ ).

Since our main objective herein relates to the generation of freak waves by the Benjamin-Feir instability and since eqs. (44.5) show good agreement with the fully nonlinear inviscid equations for the two dimensional instability diagram, we feel that many of the features of the primitive equations are retained in the GDE equation. This suggests that a study of the generation of freak waves using this higher order formulation may indeed allow us to investigate many of the effects of directional spreading on the generation of freak waves.

The GDE equations have been solved numerically using a standard pseudo-spectral code fully dealiased and with second order time-stepping on a doubly-periodic domain with a spatial discretization of $256 \times 64$. The initial conditions consist of a two-dimensional wave field characterized by the JONSWAP power spectrum. In order to statistically search for extreme wave events we focus on the temporal evolution of the fourth-order, central moment (kurtosis) of the probability density function of the wave amplitude. The kurtosis is a statistical measure of the probability density function of the wave amplitudes: the larger/wider the tails are, the larger are the value of the kurtosis. It is clear that when the kurtosis is much larger than 3 (the well-known value for a Gaussian process) we are faced, in physical space, with an extreme wave event that likely corresponds to a "freak" wave (see below for details).

In order to prepare the initial wave field it is necessary to generate a directional, frequency spectrum $S(f, \theta)=$ $P(f) G(\theta)$, where $P(f)$ is the original JONSWAP spectrum, and then to transform it into the associated wave number spectrum, $S\left(k_{x}, k_{y}\right)$. The directional spreading function $G(\theta)$ used here is a cosine-squared function in which only the first lobe (relative to the dominant wave direction) is considered:

$$
G(\theta)= \begin{cases}\cos ^{2}\left(\frac{\pi}{2 \beta} \theta\right) & \text { if }-\beta \leq \theta \leq \beta \\ 0 & \text { else }\end{cases}
$$

$\beta$ is an in important parameter that provides a measure of the directional spreading, i.e. as $\beta \rightarrow 0$ the waves become increasingly unidirectional. Using the linear dispersion relation for infinite water depth, $\omega=\sqrt{g|\mathbf{k}|}$, the wave number spectrum can be written:

$$
\left.S\left(k_{x}, k_{y}\right)=\frac{\alpha}{|\mathbf{k}|^{3}} \frac{1}{2|\mathbf{k}|} e^{-\frac{3}{2}\left(\frac{k_{0}}{|\mathbf{k}|}\right)^{2}} \gamma \exp ^{\operatorname{ex}} \frac{\left(\sqrt{|\mathbf{k}|}-\sqrt{k_{0}}\right)^{2}}{2 \sigma_{0}^{2} k_{0}^{2}}\right] G(\theta)
$$

where $\theta=\arctan \left(k_{y} / k_{x}\right)$. From eq. (7) the twodimensional surface elevation is computed in the following simple way (see [25]):

$$
\eta(x, y)=\sum_{i=1}^{N} \sum_{j=1}^{M} C_{i j} \cos \left(k_{i} x+k_{j} y+\phi_{i j}\right),
$$

where the $\phi_{i j}$ are uniformly distributed random phases on the interval $(0,2 \pi)$ and $C_{i j}=\sqrt{4 S\left(k_{i}, k_{j}\right) \Delta k_{i} \Delta k_{j}}$. Most of our numerical simulations have been started with a field of size $5026.5 \mathrm{~m} \times 1885 \mathrm{~m}$, with a dominant wave number $k_{0}=0.02 \mathrm{~m}^{-1}$, corresponding to a characteristic wavelength of $\sim 300 \mathrm{~m}$. The time step adopted is 1 second and the full duration of the simulation for each run is one hour in real time. We recall that the nonlinear time scale $T_{N L}$ for the effects of the Benjamin-Feir instability to appear is of the order of $O\left(1 /\left(\varepsilon^{2} \omega_{0}\right)\right)$. In our numerical simulations the wave steepness is $\varepsilon \sim 0.1$ and the dominant frequency $f_{0}$ can be estimated from $k_{0}$ using the linear dispersion relation in infinite water depth, $f_{0} \simeq 0.07 \mathrm{~s}^{-1}$. A rough estimation of the nonlinear time scale is $T_{N L} \simeq 250 \mathrm{~s}$. For each time step the two-dimensional surface has been visualized and the kurtosis has been computed from the space series associated with each instant in time of the complete evolution. In Fig. 1 $\mathrm{a}, \mathrm{b}$ we show that, for small values of the enhancement parameter $\gamma$ (for $\gamma=1$ the significant wave height $H_{s}$, computed as 4 times the standard deviation of the wave amplitude, was estimated to be $H_{s}=7.26 \mathrm{~m}$ ), the kurtosis is found to be $\simeq 3$ for all the evolution, independently of the value of the spreading parameter $\beta$. Additional numerical simulations, with many different sets 
of random phases, have been performed and the results are quantitatively similar to the one just presented. A more interesting case, from the point of view of extreme waves, corresponds to the numerical simulations with initial conditions characterized by $\gamma=5\left(H_{s}=9.8 \mathrm{~m}\right)$. The main results of these latter simulations are shown in Fig. $2 \mathrm{a}, \mathrm{b}$.
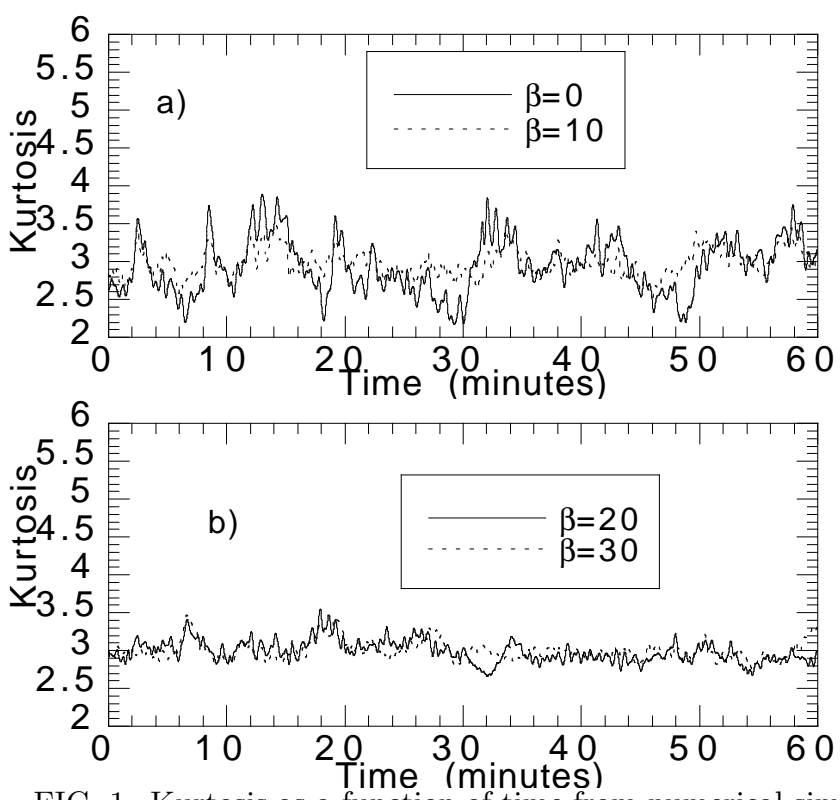

FIG. 1. Kurtosis as a function of time from numerical simulations with $\alpha=0.0081$ and $\gamma=1$, for $\beta=0,10$ (a) and $\beta=20,30$ (b) .
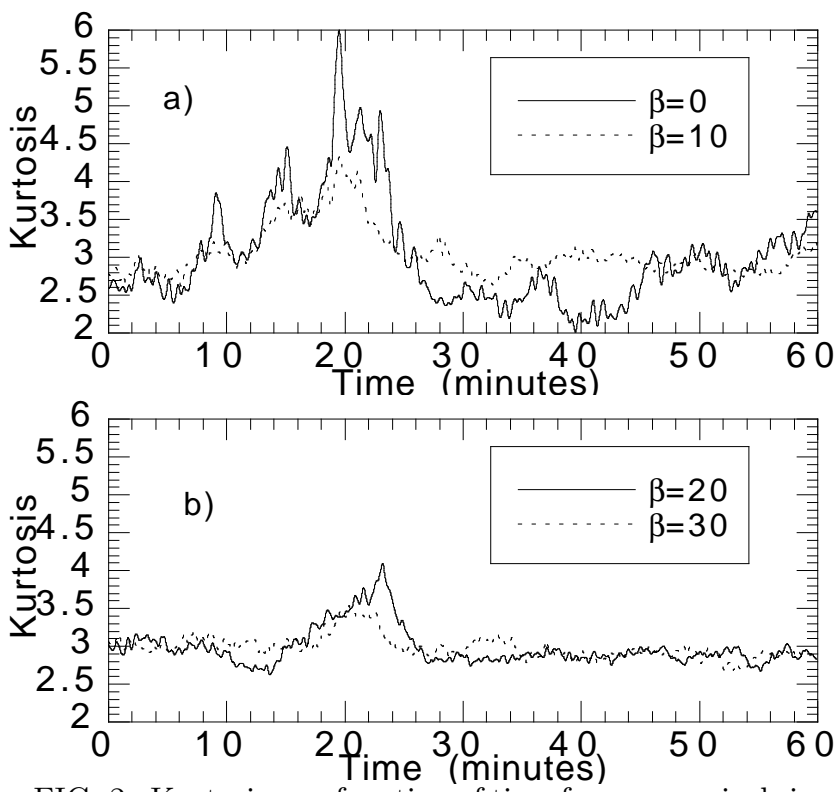

FIG. 2. Kurtosis as a function of time from numerical simulations with $\alpha=0.015$ and $\gamma=5$, for $\beta=0,10$ (a) and $\beta=20,30$ (b) .

Here we see that for small values of the spreading parameter, $\beta$, the kurtosis suddenly starts to increase (Fig.
2 a) indicating the onset of the Benjamin-Fier instability. The kurtosis is seen to grow, to reach a maximum value and then to decrease and oscillate around the value $\simeq 3$. An example of the wave field with the kurtosis $=4.3$, resulting from the evolution of an initial condition with $\gamma=5$ and $\beta=10$ is given in Fig. 3: a large amplitude wave with respect to the rest of the quasi-gaussian background appears in the middle of the two dimensional surface plot; note also that before the large wave a deep hole is also evident (this phenomenology is consistent with many descriptions of mariners who happened to run into a freak wave). As the spreading parameter, $\beta$, is increased in subsequent numerical simulations (Fig. 20 b) the kurtosis continues to grow but does not reach values as high as in the previous cases. For the larger values of $\beta$, the kurtosis oscillates around $\simeq 3$, indicating that the probability density function for the wave amplitudes is approximately Gaussian.

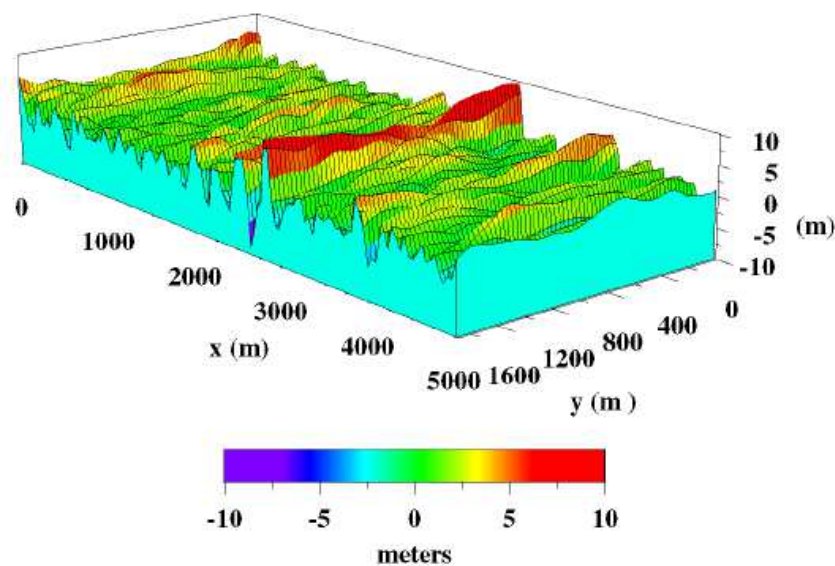

FIG. 3. A two dimensional free surface showing a quasi gaussian backgraound and a large wave amplitude inthe middle of it. The kurtosis of the field is 4.3. The surface has been obtained from the nonlinear evolution of a random phase wave field characterized by a JONSWAP spectrum with $\alpha=0.0081, \gamma=5$ and $\beta=10$.

A considerable number of numerical simulations have been carried out using different values for the selected sets of random phases. The results are in qualitative agreement with the case just discussed. However, we find that the times at which the rare events appear are different for different sets of random phases and that the maximum values of the kurtosis can be also be quite different. For a number of random realizations we have found, for the case of a quasi-unidirectional spectrum, the appearance of as many as two extreme events in a single hour of simulation time. We note that for very small directional spreading (essentially the one-dimensional case) the pattern of freak wave generation is almost periodic, primarily because of the well-known phenomenon of FermiPasta-Ulam recurrence. In the fully $2+1$ case the physics is much more complicated: as noted in [26], there is a permanent frequency downshift which excludes the pos- 
sibility of recurrence. As a final general consideration it should be mentioned that the classical statistical description of deep water wave trains (using the kinetic equations of the Hasselmann, or Boltzmann, type in the quasiGaussian approximation) is based on the assumption that the wave field is spatially homogeneous (see for example [18): under this condition the Benjamin-Feir instability is suppressed and the transfer of energy in the power spectrum is ruled only by the four-wave resonant interaction whose nonlinear time scale is $T_{N L}=O\left(1 /\left(\varepsilon^{4} f_{0}\right)\right)$. Therefore, if the statiscal prediction of the occurence of freak waves is desired, a new form of kinetic equation should be adopted. This very interesting application is, however, beyond the scope of the present paper and will be considered in a future work.

Acknowledgements The authors are grateful to D. Resio for valuable discussions at the beginning of the work. M. O. was supported by a Research Contract from the Università di Torino. This work was supported by the Office of Naval Research of the United States of America (T. F. Swean, Jr. and T. Kinder) and by the Mobile Offshore Base Program of ONR (G. Remmers). Consortium funds and Torino University funds (60\%) are also acknowledged.

[1] R. G. Dean, Water Wave Kinematics, 609-612, Kluwer, Amsterdam (1990).

[2] In Proc. Rogue Waves 2000, 29-30 November 2000 Brest, France, to be published, (2001).

[3] D. H. Peregrine, "Interaction of water waves and currents," Ad. Appl. Mech. 16, 9 (1976).

[4] I. Lavrenov, "The wave energy concentration at the Agulhas current of South Africa," Nat. Hazards 17, 117 (1998).

[5] B. S. White and B. Fornberg, "On the chance of freak waves at sea" J. Fluid Mech. 335, 113 (1998).

[6] M. C. Davis and E. E. Zarnick, "Testing ship model in transient waves," Proc. 5th int. Symp. on Naval Hydrogr., office of Naval Res, 507 (1964).

[7] K. Trulsen and K. Dysthe, "Freak Waves - A threedimensional wave simulation," Proc. 21st Symp. on Naval Hydrodynamics, National Academy Press 550 (1997).

[8] T. B. Benjamin and J. E. Feir, "The disintegration of wave trains on deep water. Part 1. Theory," J. Fluid Mech. 27, 417, (1967).

[9] B. M. Lake, H. C. Yuen, H. Rundgaldier and W. E. Ferguson, "Nonlinear deep-water waves: theory and exper- iment. Part 2. Evolution of a continuous wave train," J. Fluid Mech. 83, 49 (1977).

[10] M. P. Tulin and T. Waseda, "Laboratory observations of wave group evolution, including breaking effects," J. Fluid Mech. 378, 197 (1999).

[11] E. R. Tracy, Topics in nonlinear wave theory with applications, Ph.D. Thesis, University of Maryland (1984); E. R. Tracy and H. H. Chen, Phys. Rev. A 37, 815 (1988).

[12] H. C. Yuen, "Nonlinear Dynamics of Deep-Water Gravity Waves," Adv. Appl. Mech. 22, 67 (1982).

[13] K. L. Henderson, D. H. Peregrine and J. W. Dold, "Unsteady water wave modulation: fully nonlinear solutions and comparison with the nonlinear Schroedinger equation," Wave Motion 29 , 341 (1999).

[14] M. Onorato, A. R. Osborne and M. Serio, "Occurrence of Freak Waves from Envelope Equations in Random Ocean Wave Simulations", to be published in Rogue Wave 2000, 29-30 November 2000 Brest, France, (2001).

[15] K. Dysthe, "Note on a modification to the nonlinear Schroedinger equation for application to deep water waves," Proc. R. Soc. Lond. A 369, 105 (1979).

[16] K. Trulsen and K. Dysthe, "A modified nonlinear Schrodeinger equation for broader bandwidth gravity waves on deep water," Wave Motion 24, 417 (1996).

[17] M. Onorato, A. R. Osborne, M. Serio and S. Bertone, "Freak waves in random oceanic sea states," to be published in Phys. Rev. Lett., June (2001).

[18] Komen, G. J., Cavaleri, L., Donelan, M., Hasselman, K., Hasselman, S., Janssen, P. A. E. M.: Dynamics and modeling of ocean waves, Cambridge University Press, (1994).

[19] A. R. Osborne, M. Onorato and M. Serio, "The nonlinear dynamics of rogue waves and holes in deep water gravity wave trains," Phys. Lett. A 275, 386 (2000).

[20] Zakharov, V., "Stability of periodic waves of finite amplitude on the surface of a deep fluid," J. Appl. Mech. Tech. Phys. 9, 190 (1968).

[21] K. Trulsen, I. Kliakhandler, K. Dysthe and M. G. Velarde, "On weakly nonlinear modulation of waves on deep water," Phys. Fluids 12, 2432 (2000).

[22] V. P. Krasitskii, "On reduced equations in the Hamiltonian theory of weakly nonlinear surface waves," J. Fluid Mech. 272, 1 (1994).

[23] M. Stiassnie, "Note on the modified nonlinear Schroedinger equation for deep water waves," Wave Motion 6, 431 (1984).

[24] J. W. McLean, "Instabilities of finite-amplitude water waves," J. Fluid Mech. 114, 315 (1982).

[25] L. E. Borgman, "Techniques for Computer Simulation of Ocean Waves", in Topiccs in Ocean Physics, Proc. of the Int. School E. Fermi, Eds. A. R. Osborne and P. Malanotte Rizzoli, 373 (1980)

[26] K. Trulsen and K. B. Dysthe "Frequency downshift in three-dimensional wave trains in a deep basin," J. Fluid Mech. 352, 359 (1997). 